\title{
AOF1 is a histone H3K4 demethylase possessing demethylase activity-independent repression function
}

Ze Yang, Jun Jiang, M David Stewart, Shankang Qi, Kenichi Yamane, Jiwen Li, Yi Zhang, Jiemin Wong

Cell Research (2010) 20:858. doi:10.1038/cr.2010.90; published online 1 July 2010

Correction to: (2010) 20:276-287. doi:10.1038/cr.2010.12; published online 26 January 2010

The authors report that the name of the third author was incorrect in the article. The correct name is M David Stewart, not David M Stewart. The authors apologize for the error. 